Sharif University of Technology
Scientia Iranica
SCIENTIA
I RAN I CA

\title{
Numerical study of material properties, residual stress and crack development in sintered silver nano-layers on silicon substrate
}

\author{
M. Keikhaie ${ }^{a}$, M.R. Movahhedy ${ }^{a, *}$, J. Akbari ${ }^{a}$ and H. Alemohammad ${ }^{b}$ \\ a. Center of Excellence in Design, Robotics and Automation, Department of Mechanical Engineering, Sharif University of \\ Technology, Tehran, P.O. Box 14588-89694, Iran. \\ b. Department of Mechanical and Mechatronics Engineering, University of Waterloo, 200 University Ave West, Waterloo, Ontario, \\ N2L 3G1, Canada.
}

Received 3 January 2015; received in revised form 8 May 2015; accepted 27 June 2015

\section{KEYWORDS}

Thin film;

Silver nanoparticles;

Nanoscale sintering;

Residual stress.

\begin{abstract}
In order to improve the performance of thin film devices, it is necessary to characterize their mechanical, as well as electrical, properties. In this work, a model is developed for analysis of the mechanical and electrical properties and the prediction of residual stresses in thin films of silver nanoparticles deposited on silicon substrates. The model is based on inter-particle diffusion modeling and finite element analysis. Through simulation of the sintering process, it is shown how the geometry, density, and electrical resistance of the thin film layers are changed by sintering conditions. The model is also used to approximate the values of Young's modulus and the generated residual stresses in the thin film in the absence and presence of cracks in the film. The results are validated through comparing them with available experimental data.
\end{abstract}

(C) 2016 Sharif University of Technology. All rights reserved.

\section{Introduction}

Synthesizing the thin films of conductive materials (e.g. silver) is a critical step in the chip level manufacturing process. Off-chip interconnects in the microelectronic assembly process also rely on printed thin film interconnections. The constant need for increasing the functionality of microelectronic products, while reducing their size and weight, calls for more precise control of thin film interconnection manufacturing process parameters. In most applications, the films are very thin, in the range of nanometers, bonded to comparatively thick substrates, in the range of hundreds of micrometers. The property mismatch between the substrate and thin film can result in structural failures such as crack formation and delamination. Addition-

*. Corresponding author. Tel.: +98216616 5501; E-mail address: movahhed@sharif.edu (M.R. Movahhedy) ally, there exist applications for flexible electronics, where the films are deposited on substrates with high compliance. In these applications, the flexibility of the substrate elevates the failure modes of the deposited thin films. As a result, determining the mechanical properties of the thin film is of prime importance to gain an insight into failure modes of the printed microelectronic circuits and optimize process parameters, accordingly, to minimize potential structural failure. These effects have been explored in previous research work, both experimentally and theoretically [1-13]. Greer and Street [1,2] investigated residual stresses, Young's modulus, hardness and variation of thickness, and the density and resistivity of sintered silver nanoparticles, experimentally. Lee et al. [3] performed an experimental investigation to determine the crack pattern in silver thin films deposited on a variety of substrat with different thicknesses and widths. Mei et al. estimated the residual stresses of a sandwiched as- 
sembly using simulation and experimental research [4]. Zhou et al. modeled the thermal residual stresses of $\mathrm{ZrO}_{2} /\left(\mathrm{ZrO}_{2}+\mathrm{Ni}\right)$ sandwich ceramics using simulation and experimental research [9]. Marques et al. examined the effect of heat treatment on the microstructure and residual stress fields of a weld multilayer experimentally [10]. Wang et al. also assessed the mechanical behavior of multilayer systems in heating and cooling phases, and determined their residual stresses using FEM [11].

The manufacturing of thin films may involve the printing or spin coating of metallic nanoparticle solutions, followed by thermal sintering, in which the deposited thin films go through a heating-cooling cycle. The mechanical and electrical properties and the induced residual stress in the nanoparticle thin films are highly affected by the sintering process parameters and pre-sintering geometrical features.

In this paper, we aim to develop a modeling platform to study the sintering of silver nanoparticles based on inter-particle diffusion and finite element analysis. The model is used for estimation of the mechanical and electrical properties in silver thin films deposited on silicon substrates. The film density, thickness, Young's modulus, electrical resistance, and induced residual stress in crack-free and cracked thin films of silver nanoparticles will be studied in this paper.

\section{Inter-particle diffusion modeling}

\subsection{Sintering process}

Sintering is a processing technique to produce densitycontrolled materials and components from metal and/or ceramic powders by applying thermal energy. The sintering process involves three stages:

1. Adhesion stage (start of diffusion);

2. Initial stage (particles geometry change by neck growth);

3. Intermediate and final stage (diffusion by shrinkage).

There is no clear distinction between the stages, as the processes associated with each stage tend to overlap; however, some generalizations can be made to distinguish the different stages in a sintering process. Swinkels and Ashby [14] considered specific governing relations of micro-scale particles for each stage. The micro-scale sintering process can be extended to nanoparticles, with some modifications. For example, nano-scale particles have a significantly larger surface energy $\left(\gamma_{s}\right)$ than micro-scale particles, which results in a significant reduction in sintering temperature. Nanoparticle sintering is classified into different types based on the dominant mechanisms for shrinkage or

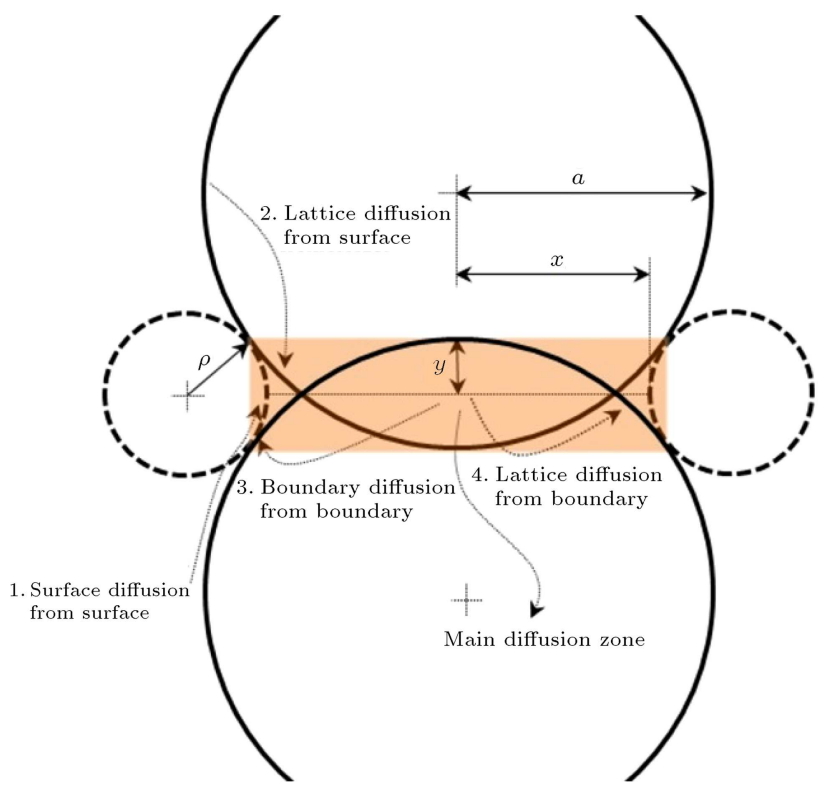

Figure 1. Schematic diagram of nanoparticles and the sintering mechanism.

Table 1. Low-temperature sintering mechanisms in polycrystalline nanoparticles.

\begin{tabular}{llc}
\hline \multicolumn{1}{c}{ Mechanism } & Diffusion path & Shrinkage \\
\hline Surface diffusion & Surface & No \\
Lattice diffusion & Surface & No \\
Grain-boundary diffusion & Grain-boundary & Yes \\
Lattice diffusion & Grain-boundary & Yes \\
\hline
\end{tabular}

densification. Agglomeration in polycrystalline materials (e.g. silver) is governed by solid state sintering, in which the major neck growth mechanisms for nanoparticles of more than $10 \mathrm{~nm}$ in size are grainboundary diffusion and surface diffusion [15]. As shown in Figure 1, the low-temperature sintering of nanoparticles involves four major diffusion mechanisms. The diffusion mechanisms and the dominant diffusion paths are listed in Table 1. The main diffusion zone (shaded area in Figure 1) is the area in which most of the geometric variations occur during agglomeration of the two nano particles.

Table 1 also summarizes the mechanisms which are important for neck formation and shrinkage in sintering nanoparticles.

\subsection{Governing equations}

In this paper, we employ modified micro-scale governing equations adapted for nanoparticle sintering to determine only an approximate configuration of particle diffusion, as follows.

\subsubsection{Adhesion stage}

To solve the differential equations of diffusion, an initial value $\left(x_{0}\right)$ is required, which is obtained from the 
adhesion equation:

$$
x_{0}=\left(\frac{\gamma_{s} a^{2}}{10 \mu}\right)^{\frac{1}{3}},
$$

where $\gamma_{s}$ is surface energy $\left(\mathrm{J} / \mathrm{m}^{2}\right)$, $a$ is particle radius $(\mathrm{m})$ and $\mu$ is shear modulus $\left(\mathrm{N} / \mathrm{m}^{2}\right)$.

\subsubsection{Initial stage}

In the initial stage, particles can be rearranged into more stable positions by rotating and sliding in response to the sintering forces. During particle rearrangement, there is an increase in inter-particle contact, which enables the formation of a neck between particles. Neck formation and growth are initiated by inter-particle diffusion. The initial stage of sintering is assumed to last until a neck radius of around 0.4 to 0.5 of the particle radius is attained [16].

The governing equations for the initial stage are as follows [14]:

a) Surface diffusion from surface source:

$$
\dot{V}_{1}=\frac{3 \pi x D_{s} \delta_{s} \gamma_{s} \Omega\left(K_{3}-K_{2}\right)}{d_{2} k T},
$$

where $\dot{V}_{1}$ is diffusive currents $\left(\mathrm{m}^{3} / \mathrm{s}\right), x$ is neck radius $(\mathrm{m}), D_{s}$ is surface diffusion coefficient $\left(\mathrm{m} / \mathrm{s}^{2}\right)$, $\delta_{s}$ is effective surface thickness $(\mathrm{m}), \Omega$ is atom volume $\left(\mathrm{m}^{3}\right), K_{2}$ and $K_{3}$ are curvatures $(1 / \mathrm{m}), d_{2}$ is a geometric variable, $k$ is Boltzmann's constant $\left(\mathrm{J} /{ }^{\circ} \mathrm{K}\right)$, and $T$ is absolute temperature.

b) Lattice diffusion from a surface source:

$$
\dot{V}_{2}=\frac{3 \pi x D_{s} \gamma_{s} \Omega\left(K_{3}-K_{m}\right)}{k T},
$$

where $\dot{V}_{2}$ is diffusive currents $\left(\mathrm{m}^{3} / \mathrm{s}\right)$ and $K_{m}$ is curvature $(1 / \mathrm{m})$.

c) Grain-boundary diffusion from a boundary source:

$$
\dot{V}_{3}=\frac{16 \pi D_{b} \delta_{b} \gamma_{s} \Omega\left(-K_{1}\right)}{2 k T},
$$

where $\dot{V}_{3}$ is diffusive currents $\left(\mathrm{m}^{3} / \mathrm{s}\right), D_{b}$ is grainboundary diffusion coefficient $\left(\mathrm{m} / \mathrm{s}^{2}\right), \delta_{b}$ is effective boundary thickness $(\mathrm{m})$, and $K_{1}$ is curvature $(1 / \mathrm{m})$.

d) Lattice diffusion from a boundary source:

$$
\dot{V}_{4}=\frac{32 \pi \rho \theta D_{v} \gamma_{s} \Omega\left(-K_{m}\right)}{2 k T},
$$

where $\dot{V}_{4}$ is diffusive currents $\left(\mathrm{m}^{3} / \mathrm{s}\right), \rho$ is radius of neck surface $(\mathrm{m}), \theta$ is geometric variable, and $D_{v}$ is lattice diffusion coefficient $\left(\mathrm{m} / \mathrm{s}^{2}\right)$.

Neck growth rate $(\dot{x}, \mathrm{~m} / \mathrm{s})$ can be obtained by combining the diffusion currents from Eqs. (1) to (5), as follows:

$$
\dot{x}=\frac{\dot{V}_{1}+\dot{V}_{2}+\dot{V}_{3}+\dot{V}_{4}}{2 \pi x \theta \rho} .
$$

\subsubsection{Intermediate and final stage}

In the intermediate and final stages of sintering, after formation of the neck, the center distance between the particles is reduced. The grain-boundary and lattice diffusion mechanism are dominant during this stage of sintering. As a result, the governing equations are [14]:

a) Grain-boundary diffusion from a boundary source:

$$
\dot{V}_{5}=-\frac{2 \pi D_{b} \delta_{b} \gamma_{s} \Omega K_{1}}{k T\left(\ln \left(\frac{x+\rho}{\rho}\right)-\frac{3}{4}\right)},
$$

where $\dot{V}_{5}$ is a diffusive current $\left(\mathrm{m}^{3} / \mathrm{s}\right)$.

b) Lattice diffusion from a boundary source:

$$
\dot{V}_{6}=-\frac{4 \pi \rho D_{v} \gamma_{s} \Omega K_{m}}{k T\left(\ln \left(\frac{x+\rho}{\rho}\right)-\frac{3}{4}\right)},
$$

where $\dot{V}_{6}$ is a diffusive current $\left(\mathrm{m}^{3} / \mathrm{s}\right)$.

The linear shrinkage rate $(\dot{y}, \mathrm{~m} / \mathrm{s})$ can be calculated using the following relation:

$$
\dot{y}=\frac{\dot{V}_{5}+\dot{V}_{6}}{\pi\left(x^{2}-\rho^{2}\right)} .
$$

Swinkels and Ashby [14] showed that $x, y, a, \rho, \theta, K_{1}$, $K_{2}, K_{3}$, and $K_{m}$ are inter-related, according to the following equations:

$$
\begin{aligned}
& \rho=\frac{x^{2}}{2(a-x)} \\
& \theta=\tan ^{-1}\left[\frac{a-y}{x+\rho}\right], \\
& K_{m}=-\frac{1}{\rho}+\frac{1}{x} \\
& K_{1}=\frac{K_{m}^{2}}{K_{2}} \\
& K_{3}=\frac{2}{a} .
\end{aligned}
$$

The constants $d_{2}$ and $K_{2}$ are obtained by defining a material property constant $(A)$ :

$$
A=\frac{D_{b} \delta_{b}}{D_{s} \delta_{s}}
$$

For $A>>1\left(A=2.58 \times 10^{13}\right.$ for silver $), d_{2}$ and $K_{2}$ can be determined from [14]:

$$
\begin{aligned}
& d_{2}=0.55 \rho \\
& K_{2}=0.8199 K_{m} \sqrt{\frac{A \rho}{x}} .
\end{aligned}
$$




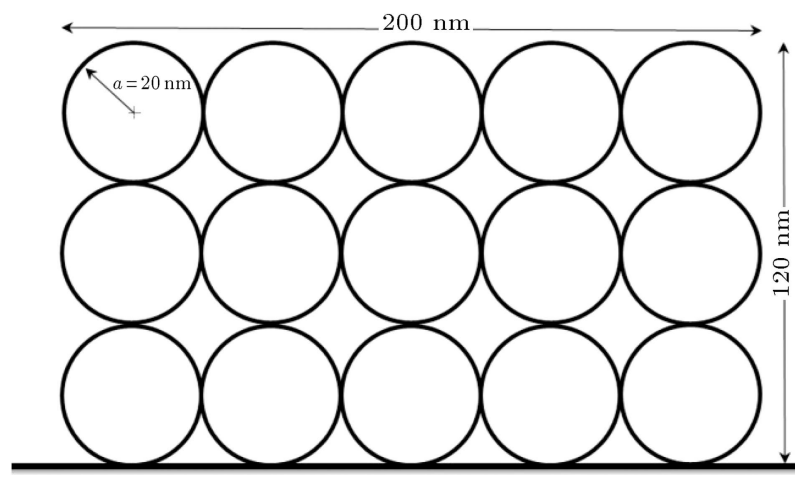

Figure 2. Schematic representation of initial film configuration before sintering process.

\subsection{Nanoparticles diffusion modeling}

For modeling the nanoparticle sintering process, we considered silver nanoparticles with a diameter of $40 \mathrm{~nm}$ arranged in a single-layer film with dimensions of $120 \times 200 \mathrm{~nm}$, as shown in Figure 2. The sintering parameters were adopted from the experimental work in $[1,2]$. According to [2], the heating-cooling cycle of the sintering process is divided into three stages:

1. Ramp-up heating from 25 to $150^{\circ} \mathrm{C}$ at a rate of $3^{\circ} \mathrm{C} / \mathrm{min}$;

2. Isothermal heating at $150^{\circ} \mathrm{C}$ for 1 hour;

3. Ramp-down cooling from 150 to $25^{\circ} \mathrm{C}$ at a rate of $3^{\circ} \mathrm{C} / \mathrm{min}$.

Since stage 2 has the maximum temperature and time, it is assumed that the major geometry changes will occur during the isothermal sintering, and the geometry changes in stages 1 and 3 are ignored. Additionally, the evaporation of organic solvent, in which the nanoparticles are suspended, is ignored, as it happens during a short period of time in stage 1 . The following further assumptions are made for modeling the diffusion:

1. Particles are pre-arranged in regular stacks, as shown in Figure 2;

2. The radius of particles remains unchanged during the sintering process;

3. The substrate is rigid and particles do not diffuse into the substrate;

4. Horizontal shrinkage in the nanoparticle layer is ignored, because the ratio of length to thickness of the layer is large.

As described in the previous section, neck formation (change in $x$ ) occurs in the initial stage of sintering, and compaction (reduction in $y$ ) happens in the intermediate and final stages of the sintering process [16].

The differential equations of diffusion in the initial, intermediate and final stages $((6)$ and $(9))$ were solved using a quasi-static approach by developing a solver code in MATLAB $^{\circledR}$ to determine neck radius $(x)$, and shrinkage $(y)$. The neck surface radius $(\rho)$, which also occurs in the differential equations, can be determined from Eq. (10). The modeling constants of silver nanoparticles for the sintering process are listed in Table A.1 in the appendix. According to Eq. (1), for $40 \mathrm{~nm}$ silver particles, the initial value $\left(x_{0}\right)$ is $2.47 \mathrm{~nm}$.

\section{Results}

\subsection{Sintered thin film geometry}

Figure 3 shows the variation of the geometrical variables during isothermal sintering at $150^{\circ} \mathrm{C}$.

From Figure 3, it is seen that the particles' shrinkage increases with a relatively constant slope, whereas the neck radius and the neck surface radius have a quick growth at first, but the slope decreases over time. This can be attributed to the fact that at the early stage of sintering, the volume of material in the neck zone is very small, compared to the volume of the particles. As a result, silver atoms flow from particles towards the neck zone. As time passes, due to the increase in the ratio of material volume in the neck zone to particle volume, the mass flow rate decreases.

A geometrical analysis was performed to extend the results of a two-particle sintering in Figure 3 to four neighboring nanoparticles (Figure 4). The analysis was

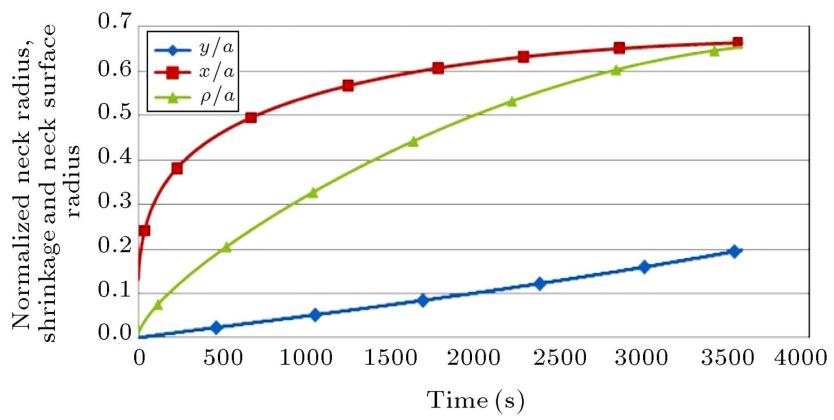

Figure 3. Normalized value of neck radius, shrinkage, and neck surface radius during isothermal sintering at $150^{\circ} \mathrm{C}$ for 1 hour.

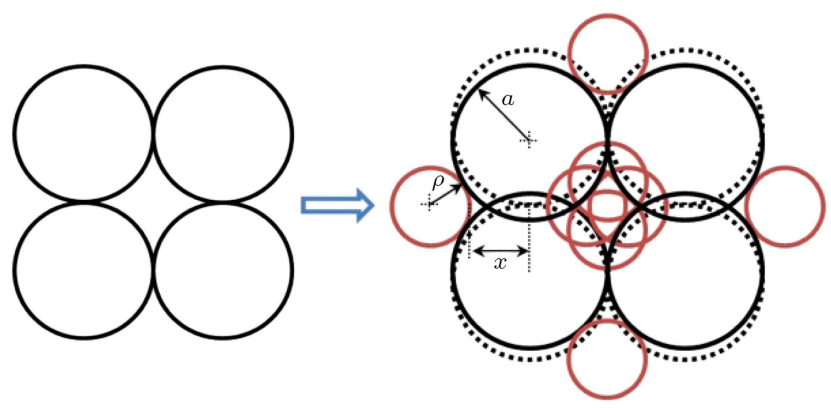

Figure 4. Initial state of four nanoparticles before sintering and the geometrical analysis to obtain the final shape after sintering. 


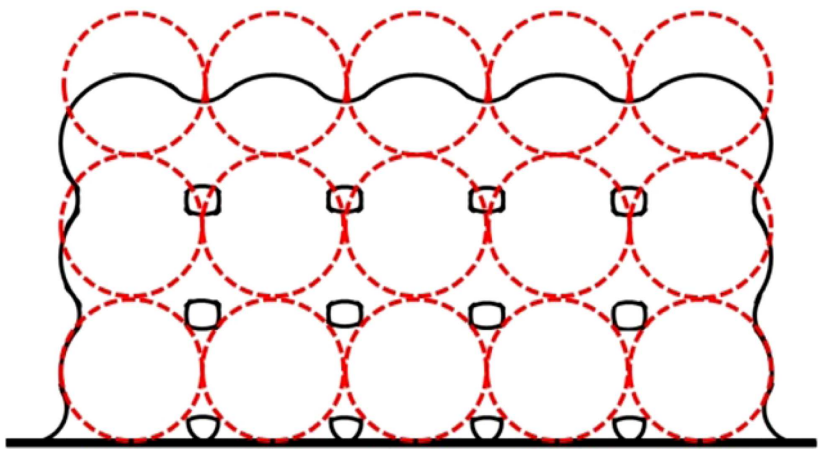

Figure 5. Nanoparticle film geometry change during sintering process at $150^{\circ} \mathrm{C}$ for 1 hour (dashed line: pre-sintering, solid line: post-sintering).

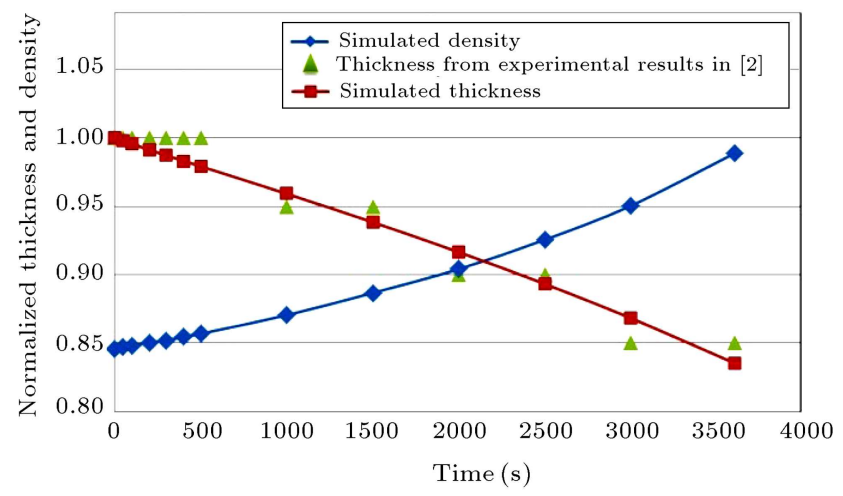

Figure 6. Normalized thickness and density changes during isothermal sintering at $150^{\circ} \mathrm{C}$ for 1 hour.

(Normalization is based on initial film thickness and bulk silver density.)

extended to the entire film to find the final geometry after sintering, as shown in Figure 5.

\subsection{Thickness and density}

Using the results of the geometrical analysis in Section 4.1, the thickness and density changes are calculated and plotted in Figure 6. As expected, the film thickness decreases, and the density increases due to the agglomeration of particles. For comparison, the thickness variation measured in an experimental work in [2] is also plotted. It is observed that the results of modeling are in good agreement with the experimental data. The results show that the film thickness decreased by $16.5 \%$ after 1 hour sintering. The initial density of the film was $84.6 \%$ of bulk silver due to the existence of voids in the particle arrangements. The density reached $98.8 \%$ of bulk state as a result of sintering.

\subsection{Electrical resistivity}

Electrical resistivity is a critical parameter for the interconnect materials. The resistance of the interconnections is directly related to the current handling capability and electrical power loss. The high electrical conductivity of the low-temperature sintered nanoscale

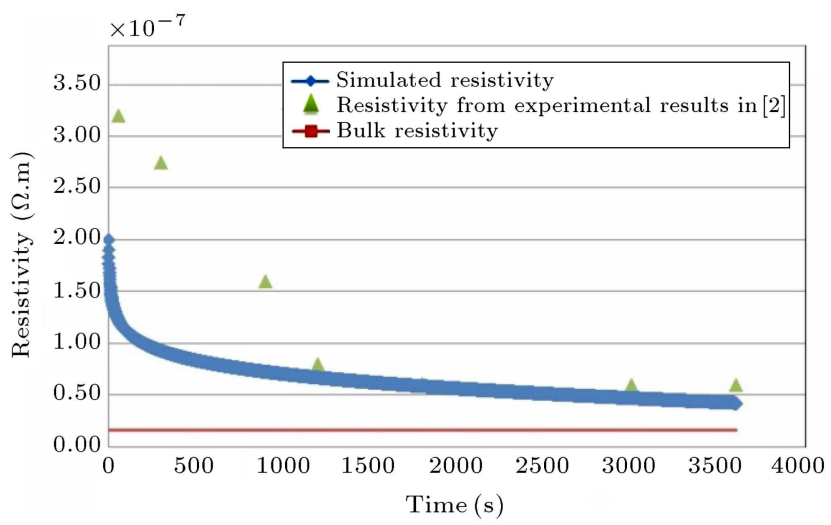

Figure 7. Resistivity changes during isothermal sintering time at $150^{\circ} \mathrm{C}$.

silver layers makes them a potentially excellent interconnecting material with high current handling capability and enhanced electrical performance. Additionally, since the low-temperature sintered silver has a relatively stable resistance up to $650^{\circ} \mathrm{C}$, it is a potentially suitable candidate for high-temperature electronic packaging applications [16].

The resistivity of the two sintered particles can be obtained from the following equation [2]:

$$
\rho(t)=\frac{2 \rho_{0}}{\pi} \ln \left\{\frac{2 a-y(t)}{y(t)}\right\},
$$

where $\rho(t)$ is resistivity $(\Omega . m)$, and $\rho_{0}$ is initial resistivity $\left(\rho_{0}=3 \times 10^{-8} \Omega\right.$.m for silver $)$.

The resistivity variation for the silver thin film, as a function of sintering time, is plotted in Figure 7 . The sintering process causes the agglomeration and densification of particles, which results in a reduction of resistivity.

As shown in the figure, at the beginning of the sintering process, the resistivity decreases with a steep slope which is due to the neck-formation through surface diffusion at the beginning of the sintering process. The resistivity values obtained from [2], for silver nanoparticle films, are plotted in the figure for comparison.

The difference between the initial values of resistivity in the model and the experimental results can be attributed to the modeling assumptions made for the arrangement of particles and by ignoring the effect of evaporating solvent. As seen, over time, the modeling results follow the experimental measurements.

\subsection{Young's modulus}

The mechanical properties of thin films are of significant importance due to their effects on the mechanical stability and functionality of the film, especially in microelectronic applications, where the thin films are exposed to temperature induced mechanical stresses. In micro-electro-mechanical devices, such as microsensors and micro-actuators, thin films are used as 
structural materials, and their functionality depends on their mechanical properties. Young's modulus is an important mechanical property of any synthesized thin film. The value of Young's modulus at bulk state is not applicable to sintered porous structures. There are several experimental methods to estimate Young's modulus. In this work, we develop a Finite Element Model (FEM) to predict Young's modulus of the sintered silver thin films.

In order to calculate Young's modulus, a finite element simulation of a tensile test is developed in ABAQUS. The modified geometry of the thin film was modeled, and the bulk modulus of silver, which is a function of temperature, is used for the solid areas. A uniform mesh with a 4-node bilinear plane stress quadrilateral (CPS4R) was used to discretize the modified film geometry. As shown in Figure 8, the filmsubstrate interface was fixed and a known tensile stress was applied on the film.

The equivalent Young's modulus was calculated using Hooke's law:

$$
E_{f}=\frac{\sigma_{f}}{\varepsilon_{f}}
$$

where $E_{f}$ is the Young's modulus of the film $(\mathrm{Pa}), \sigma_{f}$ is film stress $(\mathrm{Pa})$ and $\varepsilon_{f}$ is film strain.

Figure 9 shows the variation of Young's modulus versus temperature for the sintered and bulk state of silver. Greer et al. [2] determined the biaxial modulus

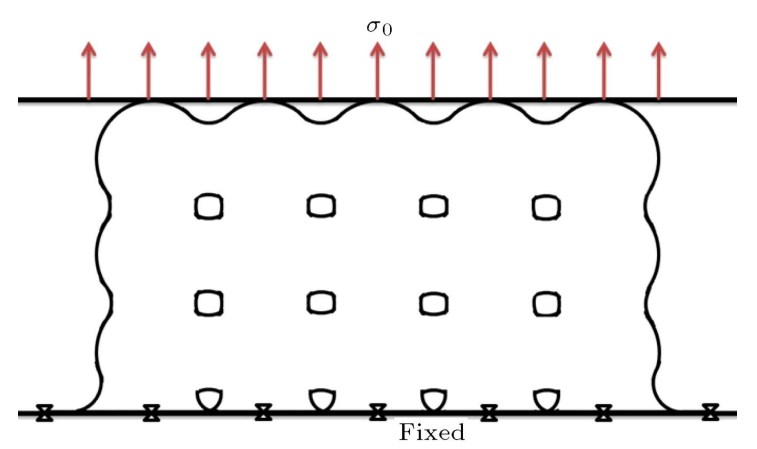

Figure 8. Modeling of tensile test in ABAQUS.

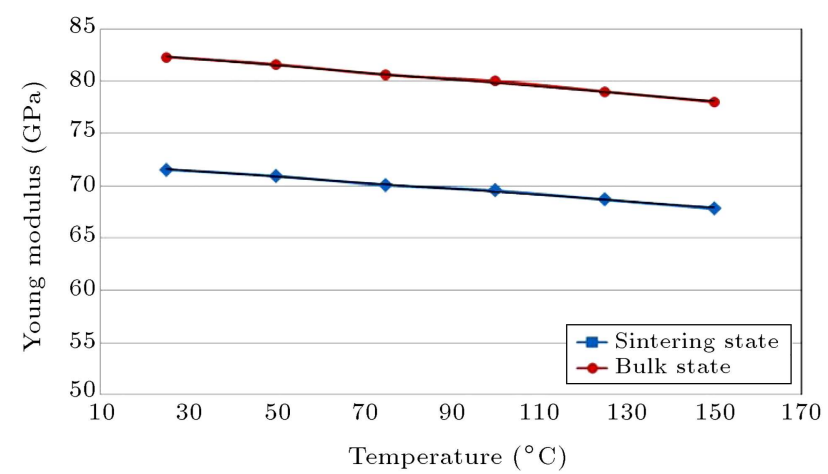

Figure 9. Young's modulus at different temperatures for sintered thin film and bulk silver. of the nanoink derived silver films by nanoindentation, and found that it is independent of the film thickness.

According to their experimental work, Young's modulus at ambient temperature $\left(25^{\circ} \mathrm{C}\right)$ was $69.3 \mathrm{GPa}$. According to Figure 9, our FEM simulation predicts a Young's modulus of $71.5 \mathrm{GPa}$ at room temperature, which is in decent agreement with the experimental results.

\subsection{Residual stress in crack-free thin film}

Residual stresses can occur through a variety of mechanisms, including plastic deformations, temperature gradients (during thermal cycle) or microstructural changes (i.e., phase transformation). During the sintering of thin films, for example, when thin film materials with different thermal and crystalline properties are deposited sequentially under different process conditions, the formation of residual stresses is inevitable. These stresses arise mainly from the evaporation of the solvent, shrinkage, and the difference between the Coefficient of Thermal Expansion (CTE) of the substrate and the film. This generates significant residual stress in the cooling phase. The stress variation through a stack of thin film materials can be very complex, and can vary between compressive and tensile stresses from layer to layer.

Hsueh [17] determined a general formulation to estimate residual stresses in thin films and substrate for a multilayered system ( $i=1$ to $n$ ), as follows:

$$
\begin{aligned}
& \sigma_{s}=\frac{2}{t_{s}^{2}}\left(3 z+2 t_{s}\right) \sum_{i=1}^{n} \frac{E_{i} t_{i}}{1-\nu_{i}}\left(\alpha_{i}-\alpha_{s}\right) \Delta T \\
& \text { for }-t_{s} \leq z \leq 0 \\
& \sigma_{i}=\frac{E_{i}}{1-\nu_{i}}\left(\alpha_{s}-\alpha_{i}\right) \Delta T \quad \text { for } i=1 \quad \text { to } n
\end{aligned}
$$

where $\sigma_{s}$ is substrate stress $(\mathrm{Pa}), \sigma_{i}$ is the $i$ th film stress $(\mathrm{Pa}), t_{s}$ is substrate thickness $(\mathrm{m}), t_{i}$ is the $i$ th film thickness $(\mathrm{m}), E_{i}$ is the Young's modulus (Pa) of the $i$ th film, $\nu_{i}$ is the $i$ th film Poisson ratio, $\alpha_{s}$ is the substrate coefficient of thermal expansion $\left(1 /{ }^{\circ} \mathrm{K}\right)$, and $\alpha_{i}$ is the $i$ th film coefficient of thermal expansion $\left(1 /{ }^{\circ} \mathrm{K}\right)$.

For a bilayer system $(n=1)$, with sub-micron thin films, the stress variation through the thickness is ignored, and the average stress is considered [18]. For a bilayer system, the substrate and average film stress are obtained from:

$$
\begin{aligned}
& \sigma_{s}=\frac{2}{t_{s}^{2}}\left(3 z+2 t_{s}\right) \frac{E_{f} t_{f}}{1-\nu_{f}}\left(\alpha_{f}-\alpha_{s}\right) \Delta T \\
& \text { for } \quad-t_{s} \leq z \leq 0 \\
& \bar{\sigma}_{f}=\frac{E_{f}}{1-\nu_{f}}\left(\alpha_{s}-\alpha_{f}\right) \Delta T
\end{aligned}
$$




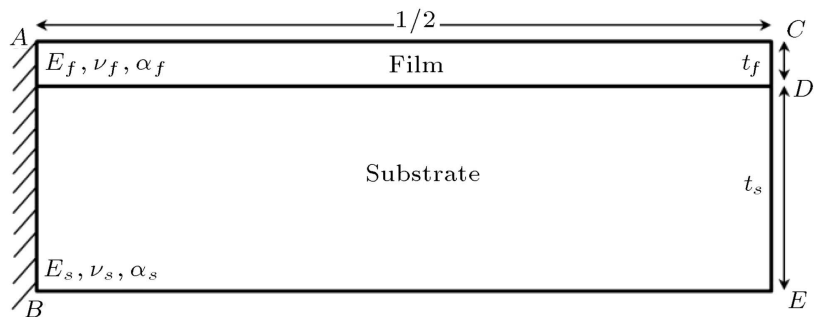

Figure 10. Schematic representation of symmetry structure of two stress-free layers with different properties.

$$
\bar{\sigma}_{f}=\left(\frac{E_{s}}{1-\nu_{s}}\right) \frac{t_{s}^{2}}{6 t_{f} r},
$$

where $\bar{\sigma}_{f}$ is average film stress $(\mathrm{Pa}), t_{f}$ is film thickness $(\mathrm{m}), \nu_{f}$ is the Poisson ratio of the film, $\alpha_{f}$ is the coefficient of thermal expansion of the film $\left(1 /{ }^{\circ} \mathrm{K}\right)$, and $r$ is substrate curvature radius $(\mathrm{m})$.

Figure 10 shows the schematic cross sectional view of a thin film deposited on a substrate. It is assumed that before the sintering process, the film is stress-free. After the sintering process, the presence of residual stress in the film causes the change of curvature in the substrate and the film. As a result, the residual stress can be estimated from the substrate curvature, according to Eq. (24).

For the thin film of silver on silicon, $\alpha_{s}<\alpha_{f}$ and $\Delta T<0$ in the cooling phase. As a result, according to Eq. (23), the residual stress in the thin film and the substrate are tension and compression, respectively (Figure 11). The location of the neutral axis is obtained from Eq. (22), when $\sigma_{s}=0$.

Distribution of residual stresses in film and substrate thickness, and also the natural axis position are schematically shown in Figure 11.

A finite element model of a silver thin film on a silicon substrate was developed in ABAQUS for estimation of residual stresses in the thin film. The model was run for thin film thicknesses of $128 \mathrm{~nm}$ and $200 \mathrm{~nm}$, and substrate width of $381 \mu \mathrm{m}$. It is assumed that the two-layer system is homogenous, and the residual stress has not caused the formation of any cracks in the thin film. To calculate deflection, using the symmetry of the film/substrate structure, half of the structure is modeled, and symmetric boundary conditions were applied at the middle by fixing that,

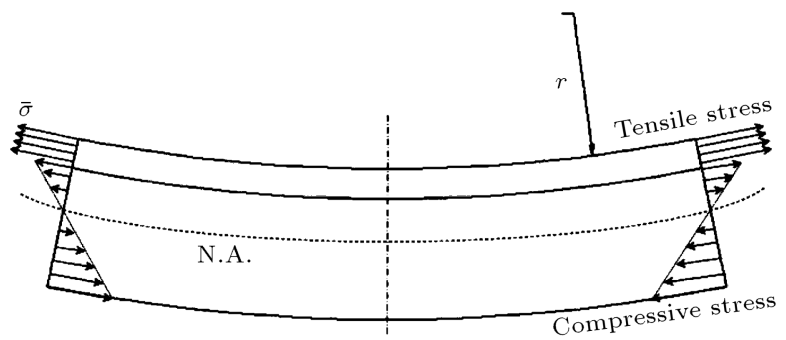

Figure 11. Schematic representation of residual stress distribution in film and substrate. while one side is free (Figure 10), and a uniform thermal loading of $150^{\circ} \mathrm{C}$ to $25^{\circ} \mathrm{C}$ was applied.

The two-layer model was meshed with a 4 -node plane stress [4] thermally coupled quadrilateral, bilinear displacement and temperature (CPS4T) mesh.

The properties of silver (Table A.2) and silicon (Table A.3) were used for the substrate and the thin film, respectively.

Figure 12 shows the geometrical changes of the bilayer system with $200 \mathrm{~nm}$ and $128 \mathrm{~nm}$ silver thin films in a cooling cycle from $150^{\circ}$ to $15^{\circ}$. By combining the geometrical results and Eq. (24), the residual stress in the film is calculated as plotted in Figure 13. The initial residual stress at $150^{\circ} \mathrm{C}$ for both thicknesses was obtained from experimental results [1] (i.e., $110 \mathrm{MPa}$ for $128 \mathrm{~nm}$ film and $60 \mathrm{MPa}$ for $200 \mathrm{~nm}$ film). As shown in the figure, the formation of the residual stress is in decent agreement with the experimental data. It is also observed that higher residual tensile stresses $(\sim 190 \mathrm{MPa})$ form in the thinner $(128 \mathrm{~nm})$ film.

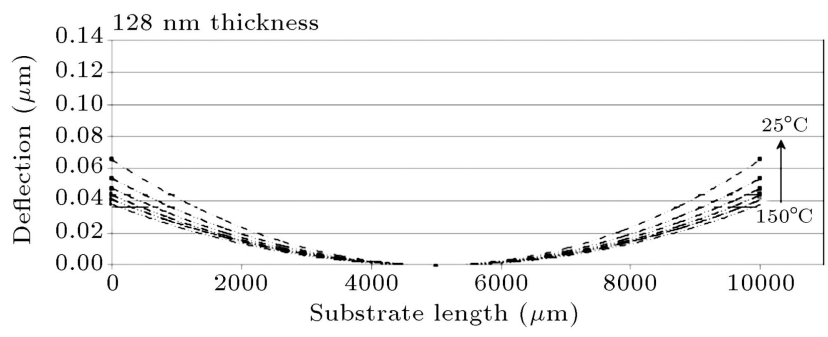

(a)

$0.14200 \mathrm{~nm}$ thickness

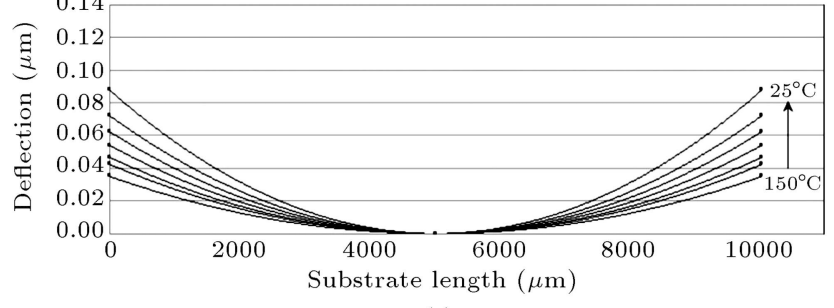

(b)

Figure 12. Deflections of film in $128 \mathrm{~nm}$ (a) and $200 \mathrm{~nm}$ (b) thickness in various temperatures from $150^{\circ} \mathrm{C}$ to $25^{\circ} \mathrm{C}$ (cooling phase).

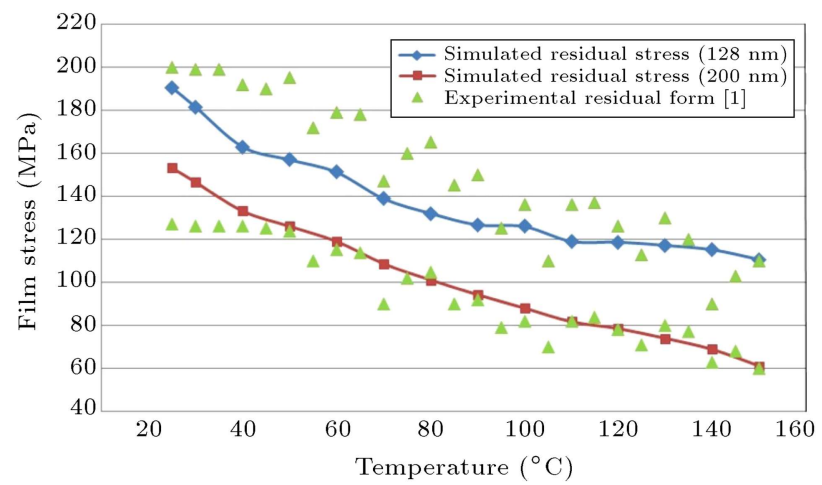

Figure 13. Stresses development in $128 \mathrm{~nm}$ and $200 \mathrm{~nm}$ silver thin films during cooling cycle. 


\subsection{Residual stress in a cracked thin film}

The effect of residual stress on crack propagation is of great practical significance and has, therefore, been the focus of much research. Considering the reliability of the components containing residual stress, it is important to determine crack growth under a residual stress field. The assumption of a crack-free thin film might not seem to be very realistic. As a result, we extend the former analysis to a cracked film. We considered a uniform elastic film of thickness $t_{f}$ bonded to the surface of an elastic substrate of thickness, $t_{s}$. The film supports a biaxial tensile mismatch stress, but is constrained to deform in plane strain bending.

It was assumed that a periodic array of parallel cracks with spacing $p$ has been formed in the film, with the crack edges being parallel to the bending axis (Figure 14). As a result of crack formation, the curvature of the substrate midplane will be reduced from the value obtained from Eq. (24). The objective is to estimate the change in curvature due to crack formation. This was carried out at the same level of approximation as the approach of the Stoney formula (Eq. (24)), but implementation of the assumptions requires somewhat more care in the present case. The main assumptions exploited here are:

(i) The state of stress in the film is determined solely by elastic mismatch of the film with the undeformed substrate, and is not significantly altered by deformation of the substrate, as it becomes curved;

(ii) The flexural stiffness of the substrate is not significantly influenced by the presence of the film material on its surface.

Under these circumstances, the change in curvature from [19] is:

$$
\frac{1}{r_{w c}}-\frac{1}{r_{n c}}=\frac{-l}{p \cdot r_{n c}} \tanh \left(\frac{p}{l}\right),
$$

where $r_{w c}$ is the film curvature radius with crack $(\mathrm{m})$, $r_{n c}$ is the film curvature radius without $\operatorname{crack}(\mathrm{m}), p$ is crack spacing, and $l$ is a length parameter that is proportional to $t_{f}$.

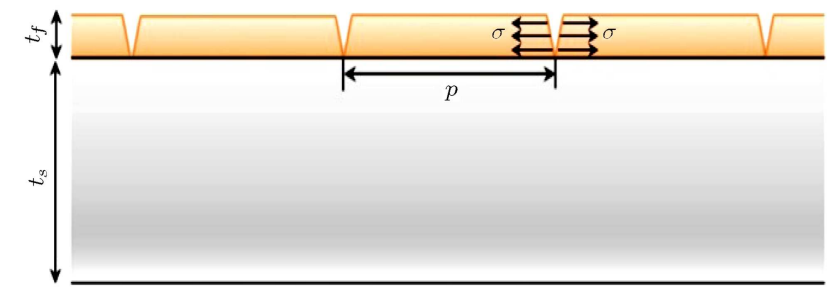

Figure 14. Schematic view of film/substrate configurations for a periodic array of parallel cracks with crack spacing $p$.
This approximation is obtained for the case of a periodic array of cracks under plane strain conditions with normal stress, $\sigma$, applied to the crack faces that tend to open the cracks. The dependence of $l$ on elastic constants can be expressed in terms of the two dimensionless parameters defined by:

$$
\begin{aligned}
& \alpha=\frac{\bar{E}_{f}-\bar{E}_{s}}{\bar{E}_{f}+\bar{E}_{s}} \\
& \beta=\frac{1}{4} \frac{\bar{E}_{f}\left(1-v_{f}\right)\left(1-2 v_{s}\right)-\bar{E}_{s}\left(1-v_{s}\right)\left(1-2 v_{f}\right)}{\bar{E}_{f}\left(1-v_{f}\right)\left(1-2 v_{s}\right)+\bar{E}_{s}\left(1-v_{s}\right)\left(1-2 v_{f}\right)},
\end{aligned}
$$

where $\alpha$ and $\beta$ are known as the Dundurs parameters [20], and:

$$
\bar{E}=\frac{E}{1-\nu^{2}}
$$

In this case, Dundurs parameters have been calculated as:

$$
\alpha=-0.26, \quad \beta=-0.0174 \text {. }
$$

With these values, $l / t_{f}=3.4$ has been chosen from [19]. Therefore, Eq. (25) is revised as:

$$
\frac{r_{n c}-r_{w c}}{r_{w c}}=-3.4 \frac{t_{f}}{p} \tanh \left(\frac{1}{3.4 \frac{t_{f}}{p}}\right) .
$$

Representative results for the ratio of substrate curvature change, due to film cracking at the mismatch stress to the corresponding curvature for a crack-free film at the same level of mismatch stress, are shown in Figure 15.

It is observed in Figure 15 that when the crack spacing is small, compared to film thickness $\left(t_{f} / p \rightarrow\right.$ $\infty)$, the crack has a large effect on stress relaxation, whereas, when the crack spacing is very large, compared to film thickness $\left(t_{f} / p \rightarrow 0\right)$, the crack has no great influence in stress relaxation, and $r_{w c}=r_{n c}$.

The squared line $\left(t_{f} / p=0\right)$ in Figure 16 shows the stress in the $588 \mathrm{~nm}$ layer calculated, using FEM

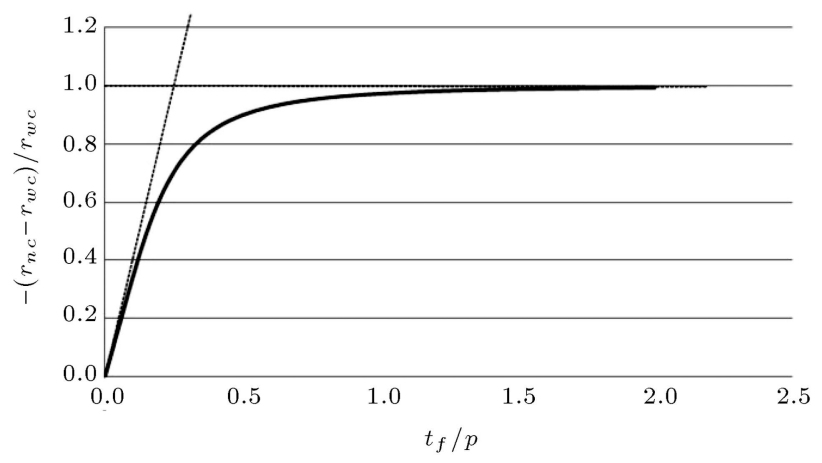

Figure 15. Ratio of change in curvature in a cracked film to curvature for a crack-free film at the same level of mismatch stress versus crack spacing $p$. 


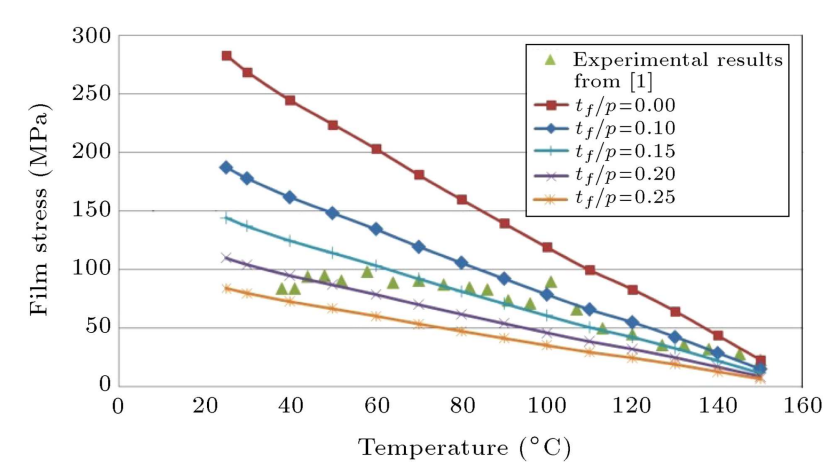

Figure 16. Stress development upon cooling for $588 \mathrm{~nm}$ film for different ratios of crack spacing.

for the crack free case, in comparison to the experimental results. It is obvious that there is significant difference between the experimental and simulation results. This difference can be attributed to crack initiation and propagation across the thin layer during the sintering process, which has not been considered in the simulation process. If the presence of cracks is considered, the stresses will be different. Figure 16 also shows the stress obtained from Eq. (24) versus sintering temperature for various values of crack spacing. It is observed that the experimental results are located at a range of different values of $t_{f} / p$ ratio. This change can be obvious, because, as the stress in the thin layer increases, the number of cracks increases and crack spacing decreases; consequently, more stress relaxation occurs, which leads to smaller net stresses.

\section{Conclusion}

In this paper, we developed a modeling platform for the analysis of electrical and mechanical properties of thermally sintered nanosilver thin films. The model was applied to $40 \mathrm{~nm}$ silver particles deposited on a silicon substrate. The results were presented for $150^{\circ} \mathrm{C}$ isothermal sintering of $128 \mathrm{~nm}$ and $200 \mathrm{~nm}$ thin films. The modeling results were also compared to the experimental test results for the same sintering conditions. The comparison shows reasonable agreement between the experimental and modeling results, which validates the model.

The model was extended for analysis of residual stress in silver thin films, which was in decent agreement with experimental data. This modeling platform can be applied for the design of sintering processes for nanoparticle thin films in micro-electromechanical and microelectronics industries, in order to develop thin films with desired qualities.

\section{References}

1. Greer, J.R. and Street, R.A. "Mechanical characterization of solution-derived nanoparticle silver ink thin films", J. Applied Physics, 101, p. 103529 (2007).

2. Greer, J.R. and Street, R.A. "Thermal cure effects on electrical performance of nanoparticle silver inks", Acta Materialia, 55, pp. 6345-6349 (2007).

3. Lee, D.J., Oh, J.H. and Bae, H.S. "Crack formation and substrate effects on electrical resistivity of inkjetprinted Ag lines", Materials Letters, 64, pp. 1069-1072 (2010).

4. Mei, Y., Chen, G., Lu, G. and Chen, X. "Effect of joint sizes of low-temperature sintered nano-silver on thermal residual curvature of sandwiched assembly", International Journal of Adhesion \& Adhesives, 35, pp. 88-93 (2012).

5. Fang, W. "Determination of the elastic modulus of thin film materials using self-deformed micromachined cantilevers", J. Micromech. Microeng., 9, pp. 230-235 (1999).

6. Murali, K.R. and Venkatachalam, K. "Electrical properties of sintered $\mathrm{CdS}_{x} \mathrm{Se}_{1-x}$ films", Chalcogenide Letters, 5(9), pp. 181-186 (2008).

7. Jung, Y. and Lawn, B.R. "Evaluation of elastic modulus and hardness of thin films by nanoindentation", $J$. Mater. Res., 19(10), pp. 3076-3080 (2004).

8. Wang, C., Cheng, L. and Zhao, Z. "FEM analysis of the temperature and stress distribution in spark plasma sintering: Modelling and experimental validation", Computational Materials Science, 49, pp. 351362 (2010).

9. Zhou, W., Zhang, R., Ai, S., Pei, Y. and Fang, D. "Analytical modeling of thermal residual stresses and optimal design of $\mathrm{ZrO}_{2} /\left(\mathrm{ZrO}_{2}+\mathrm{Ni}\right)$ sandwich ceramics", Ceramics International, 41, pp. 8142-8148 (2015).

10. Marques, M.J., Ramasamy, A., Batista, A.C., Nobre, J.P. and Loureiro, A. "Effect of heat treatment on microstructure and residual stress fields of a weld multilayer austenitic steel clad", Journal of Materials Processing Technology, 222, pp. 52-60 (2015).

11. Wang, H.J., Deng, H.A., Chiang, S.Y., Su, Y.F. and Chiang, K.N. "Development of a process modeling for residual stress assessment of multilayer thin film structure", Journal of Thin Solid Films, 584, pp. 146153 (2015).

12. Li, Kaibin, Li, D., Liu, D., Pei, Guangyu and Sun, L. "Microstructure evolution and mechanical properties of multiple-layer laser cladding coating of $308 \mathrm{~L}$ stainless steel", Applied Surface Science, 340, pp. 143-150 (2015).

13. Chou, T., Yang, S. and Chiang, K. "Overview and applicability of residual stress estimation of filmsubstrate structure", Thin Solid Films, 519, pp. 78837894 (2011).

14. Swinkels, F.B. and Ashby, M.E. "A second report on sintering diagrams", Acta Metallurgica, 29, pp. 259281 (1981).

15. Pan, H., Ko, S.H. and Grigoropoulos, C.P. "The solidstate neck growth mechanisms in low energy laser 
Table A.1. Silver properties for diffusion equations.

\begin{tabular}{cllcll}
\hline No. & Property & Value & No. & Property & Value \\
\hline 1 & $D_{b}\left(\mathrm{~m}^{2} / \mathrm{s}\right)[21]$ & $1.2 \times 10^{-5} \exp \left(-\frac{90000}{R T}\right)$ & 7 & $\mu(\mathrm{MPa})[14]$ & 26400 \\
2 & $D_{v}\left(\mathrm{~m}^{2} / \mathrm{s}\right)[21]$ & $4.4 \times 10^{-5} \exp \left(-\frac{185400}{R T}\right)$ & 8 & $\delta_{b}(\mathrm{~m})[14]$ & $5 \times 10^{-10}$ \\
3 & $D_{s}\left(\mathrm{~m}^{2} / \mathrm{s}\right)[21]$ & $5 \times 10^{3} \exp \left(-\frac{266100}{R T}\right)$ & 9 & $\delta_{s}(\mathrm{~m})[14]$ & $3 \times 10^{-10}$ \\
4 & $T_{m}\left({ }^{\circ} \mathrm{K}\right)[22]$ & 1234 & 10 & $k\left(\mathrm{~J} /{ }^{\circ} \mathrm{K}\right)$ & $1.380 \times 10^{-23}$ \\
5 & $\gamma_{s}\left(\mathrm{~J} / \mathrm{m}^{2}\right)[2]$ & 10 & 11 & $R\left(\mathrm{~J} / \mathrm{mol} .{ }^{\circ} \mathrm{K}\right)$ & 8.314472 \\
6 & $\Omega\left(\mathrm{m}^{3}\right)$ & $1.71 \times 10^{-29}$ & & & \\
\hline
\end{tabular}

Table A.2. Mechanical properties of silver.

\begin{tabular}{llcccccccc}
\hline \multicolumn{10}{c}{ Ag properties in sintering state } \\
\hline 1 & Temperature $\left({ }^{\circ} \mathrm{K}\right)$ & 290 & 300 & 320 & 340 & 360 & 380 & 400 & 420 \\
2 & CTE $\left(\frac{1}{\circ} \mathrm{K}\right) \times 10^{-6}[23]$ & 19.1 & 19.2 & 19.4 & 19.6 & 19.8 & 20 & 20.2 & 20.5 \\
3 & Young's modulus $(\mathrm{GPa})[23]$ & 71.5 & 71.4 & 70.8 & 70.2 & 69.6 & 69 & 68.4 & 67.9 \\
4 & Poisson's ratio & 0.37 & 0.37 & 0.37 & 0.37 & 0.37 & 0.37 & 0.37 & 0.37 \\
\hline
\end{tabular}

Table A.3. Mechanical properties of silicon.

\begin{tabular}{llcccccccc}
\hline \multicolumn{10}{c}{ Si properties in bulk state } \\
\hline 1 & Temperature $\left({ }^{\circ} \mathrm{K}\right)$ & 290 & 300 & 320 & 340 & 360 & 380 & 400 & 420 \\
2 & CTE $\left(\frac{1}{\circ}\right) \times 10^{-6}[24]$ & 2.52 & 2.64 & 2.8 & 2.94 & 3.05 & 3.15 & 3.25 & 3.34 \\
3 & Young's modulus $(\mathrm{GPa})$ & 130 & 130 & 130 & 130 & 130 & 130 & 130 & 130 \\
4 & Poisson's ratio & 0.28 & 0.28 & 0.28 & 0.28 & 0.28 & 0.28 & 0.28 & 0.28 \\
\hline
\end{tabular}

sintering of gold nanoparticles - a molecular dynamics simulation study", Journal of Heat Transfer, 130, 092404-1 (2008).

16. Bai, G. "Low-temperature sintering of nanoscale silver paste for semiconductor device interconnection", Ph.D. Thesis, Virginia Polytechnic Institute and State University, Blacksburg (2005).

17. Hsueh, C.H. and Paranthaman, M. "Analytical modeling of residual stresses in multilayered superconductor systems", J. Mater. Sci., 43, pp. 6223-6232 (2008).

18. Hsueh, C.H. "Modeling of elastic deformation of multilayers due to residual stresses and external bending", J. Applied Physic, 91(12), pp. 9652-9656 (2002).

19. Freund, L.B., Thin Film Materials Stress, Defect Formation and Surface Evolution, Division of Engineering, Brown University, p. 212 (2003).

20. Dundurs, J. "Edge-bonded dissimilar orthogonal elastic wedges", Journal of Applied Mechanics, 36, pp. 650-652 (1969).

21. Lee, W.K., Eadie, R.L., Weatherly, G.C. and Aust, K.T. "A study of the sintering of spherical silver powder-II. The initial stage", Acta Materialia, 26, pp. 1837-1843 (1978).

22. Perez, D.P., Silver Nanoparticles, In-Teh, p. 9 (2010).

23. Smith, D.R. and Fickett, F.R. "Low-temperature properties of silver", J. Research of the National Institute of Standards and Technology, 100(2) (1995).
24. Okada, Y. and Tokumaru, Y. "Precise determination of lattice parameter and thermal expansion coefficient of silicon between 300 and 1500 K", J. Applied Physics, 56(2), pp. 314-320 (1984).

\section{Appendix}

Diffusion coefficients and mechanical properties of Silver are given in Tables A.1 and A.2, respectively. Also, the mechanical properties of Silicon substrate are reported in Table A.3.

\section{Biographies}

Mahdi Keikhaie received his BS degree from the Department of Mechanical Engineering at Sistan and Baluchistan University, Zahedan, Iran, in 2011, and his MS degree from Sharif University of Technology, Tehran, Iran, in 2013. His research interests include NEMS and MEMS systems (sensors, actuators, etc.), fracture mechanics and CAD/CAM/CAE software.

Mohammad Reza Movahhedy received his BS degree from the University of Tehran, Iran, in 1988, his MS degree from the University of Waterloo, Canada, in 1995, and his $\mathrm{PhD}$ degree from the University of British Columbia, Canada, in 2000, all in Mechanical Engineering. He is currently Professor of Manufactur- 
ing in the Department of Mechanical Engineering at Sharif University of Technology, Tehran, Iran.

Javad Akbari received his BS degree from the School of Mechanical Engineering at Sharif University of Technology, Tehran, Iran, in 1985, his MS degree from Utsunomiya University, Japan, in 1990, and his PhD degree from Chiba University, Japan, in 1993. From 1993-1995, he worked in Seiko Seiki (Seiko Instruments Inc.). He became Associate Professor in Tokyo Institute of Technology in 2000, and is currently Associate Professor in Sharif University of Technology, Tehran, Iran. Dr. Akbari was also Visiting Professor in the Department of Mechanical Engineering at the University of Malaya from 2012 to 2014.

Hamid Alemohammad holds a PhD degree in Mechanical Engineering from the University of Waterloo, Canada, and has several years of academic and industrial experience in measurement systems, instrumentation and sensors, mechatronics systems, and advanced manufacturing. He is currently affiliated with AOMS Technologies Inc. and the University of Waterloo in Canada. He has served as reviewer and Editorial Board member for a number of academic journals and industry-academia collaboration programs. 